Reprod. Nutr. Dévelop., 1988, 28 (6 B), 1753-1762

\title{
Protéines spécifiques de la gestation chez les ruminants
}

\author{
P. HUMBLOT
}

Union Nationale des Coopératives d'Elevage et d'Insémination Artificielle, 13, rue Jouët. B.P. 65, 94703 Maisons-Alfort, France.

\section{Summary. Pregnancy specific proteins in ruminants.}

The bovine conceptus produces numerous signals during early pregnancy but at the present time only one protein (PSPB ; pregnancy specific protein B) is detectable in the peripheral blood during the first 30 days of pregnancy.

Validation criteria of the RIA for bovine PSPB have been described by Sasser et al. (1986). This review presents the results obtained in ruminants with this assay.

In the bovine PSPB concentrations rise continuously during pregnancy. This protein is found in all pregnant animals at 30 days post Al. Among cows with late embryonic mortality (i.e. with high progesterone concentrations on day $21-24$ ) only $30 \%$ present detectable PSPB concentrations between 24 and 30 days post Al. When Al are performed before 70 days post-partum, residual post- partum PSPB concentrations (from the previous pregnancy) the day of Al lowers the accuracy of positive results when PSPB RIA is used as a pregnancy test. In sheep and goat the PSPB profiles throughout pregnancy are very similar to the profile observed in the cattle. In these species the PSPB RIA may be used as a pregnancy test after 26 days post $\mathrm{Al}$

In ruminants, measurement of both progesterone and PSPB in peripheral blood plasma or serum can be very useful to study the way in which various factors may chronologically affect embryonic mortality.

L'embryon de ruminant produit précocement de nombreux signaux protéiques dont certains déterminent le maintien du corps jaune de gestation.

En effet l'action antilutéolytique d'extraits de trophoblastes (brebis, Martal et al., 1979 ; vache, Northey et French, 1980 ; Humblot et Dalla Porta, 1984) ou plus précisément de protéines trophoblastiques (brebis, Godkin et al., 1982, 1984 ; vache, Knickerbocker et al., 1986) a été clairement montrée.

Des protéines immunosuppressives ont également été mises en évidence dans les milieux de culture d'embryons âgés de 16 jours (brebis, Segerson, 1981) à 18 jours (vache, Segerson et al., 1984 ; Fisher et al., 1985).

Cependant ces signaux n'ont pas été identifiés jusqu'à présent dans la circulation périphérique. Ils ne permettent donc pas d'estimer la présence d'un embryon ni sa viabilité et ne peuvent être utilisés à des fins de diagnostic de gestation ou pour indiquer une mortalité embryonnaire.

Récemment, une protéine appelée PSPB (pregnancy specific protein B), produite par les cellules binucléées du trophoblaste a été isolée à partir d'embryons bovins âgés de 25 à 40 jours (Butler et al., 1982). Un dosage 
radioimmunologique a été mis au point et a permis la détermination des concentrations sériques de cette protéine au cours de la gestation chez les bovins (Sasser et al., 1986) ovins, caprins (Houston et al., 1986 ; Ruder et al., 1988) et cervidés (Wood et al., 1986). Cette revue présente les données acquises à l'aide du dosage radioimmunologique de cette protéine.

Concentrations de PSPB dans la circulation périphérique pendant la gestation et le post-partum.

Chez les bovins, l'évolution des concentrations sériques de PSPB au cours de la gestation a été décrite par Sasser et al. (1986). Au tout début de la gestation, le moment auquel la PSPB est détectée pour la première fois varie beaucoup d'un individu à l'autre. Ceci explique que la proportion d'animaux gestants chez lesquels la PSPB est détectable augmente régulièrement de $\mathrm{J} 15$ à $\mathrm{J} 30$ (tabl. 1). Ensuite les concentrations sériques inférieures à $1 \mathrm{ng} / \mathrm{ml}$ avant 30 jours augmentent jusqu'à la parturition pour atteindre des valeurs parfois même supérieures à $700 \mathrm{ng} / \mathrm{ml}$. Les concentrations plasmatiques aux $30^{\mathrm{e}}$ et $70^{\mathrm{e}}$ jours de gestation respectivement de $2 \mathrm{ng} / \mathrm{ml}$ et de $4 \mathrm{ng} / \mathrm{ml}$ environ (fig. 1) sont tout à fait semblables aux concentrations sériques rapportées par Sasser et al. (1986).

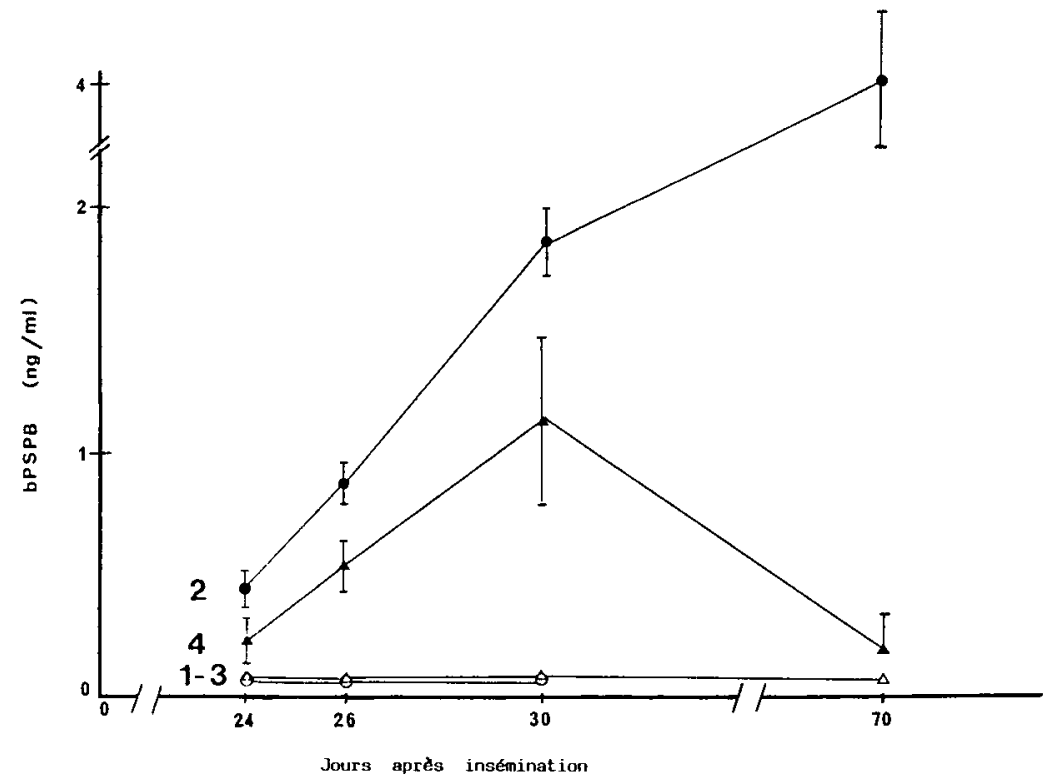

FIG. 1. - Concentrations plasmatiques de PSPB chez des femelles (1) ayant présenté une mortalité embryonnaire précoce ou une non-fécondation (2) gestantes et une mortalité embryonnaire tardive sans (3) ou avec sécrétion de PSPB (4), (selon Humblot et al., 1988a).

Au cours du post-partum, une première étude (Ruder et Sasser, 1986) avait montré l'existence d'une sécrétion résiduelle importante de PSPB. Ces données ont été confirmées par une étude où les dosages de PSPB ont été réalisés 


\section{TABLEAU 1}

Pourcentages de vaches chez lesquel/es la PSPB est détectée dans la circulation périphérique entre le $15^{e}$ et le $30^{e}$ jour de gestation (N : nombre de gestations).

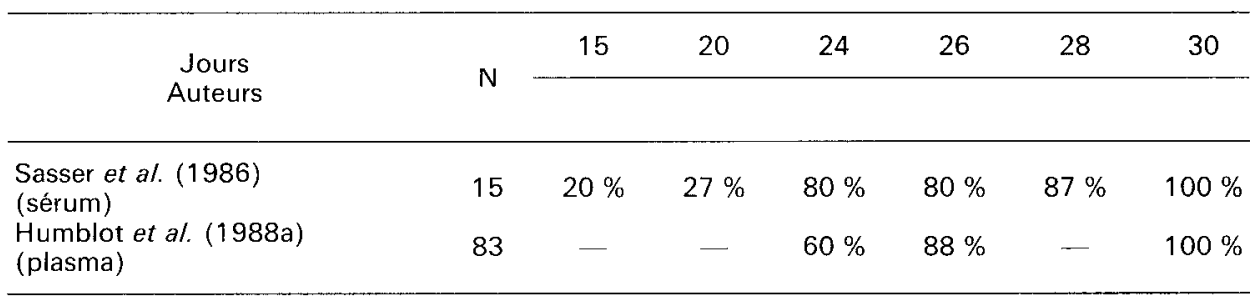

systématiquement le jour de la première insémination (Humblot et al., 1988b). Le tableau 2 illustre la régression de la moyenne des concentrations de PSPB trouvées le jour de I'IA en fonction de l'intervalle vêlage- $1^{\text {re }}$ IA. Les concentrations chutent de $21,7 \mathrm{ng} / \mathrm{ml}$ au $40^{\mathrm{e}}$ jour à $1,2 \mathrm{ng} / \mathrm{ml}$ entre 71 et 80 jours post-partum et diminuent encore jusqu'au $100^{\circ}$ jour après vêlage.

\section{TABLEAU 2}

Concentrations moyennes en bPSPB le jour de I'lA en fonction de l'intervalle vêlage $1^{\text {re }} / A$ (selon Humblot et al., 1988b).

\begin{tabular}{lcc}
\hline $\begin{array}{l}\text { Intervalle vêlage-IA } \\
\text { (jours) }\end{array}$ & Nb vaches & $\begin{array}{c}\text { Concentrations moyennes } \\
( \pm \text { s.d.) }\end{array}$ \\
\hline 40 & 1 & $21,7 \pm 3,7$ \\
$41-50$ & 25 & $10,1 \pm 6,3$ \\
$51-60$ & 30 & $6,3 \pm 5,3$ \\
$61-70$ & 31 & $2,7 \pm 2,9$ \\
$71-80$ & 19 & $1,2 \pm 0,8$ \\
$81-90$ & 21 & $0,6 \pm 0,3$ \\
$91-100$ & 15 & $0,7 \pm 0,3$ \\
$>100$ & 27 & $0,5 \pm 0,2$ \\
\hline Total & 177 & \\
\hline
\end{tabular}

Chez les caprins un profil de ce type (Humblot et al., non publié) a été réalisé en utilisant un anticorps anti-PSPB ovine (fig. 2). On observe une élévation significative des concentrations plasmatiques de PSPB à partir du $25^{\mathrm{e}}$ jour post IA chez les femelles gestantes. Les concentrations moyennes dans ce système de dosage atteignent $40 \mathrm{ng} / \mathrm{ml}$ juste avant la mise bas. La régression des concentrations au cours du post-partum semble plus rapide que chez les bovins. 


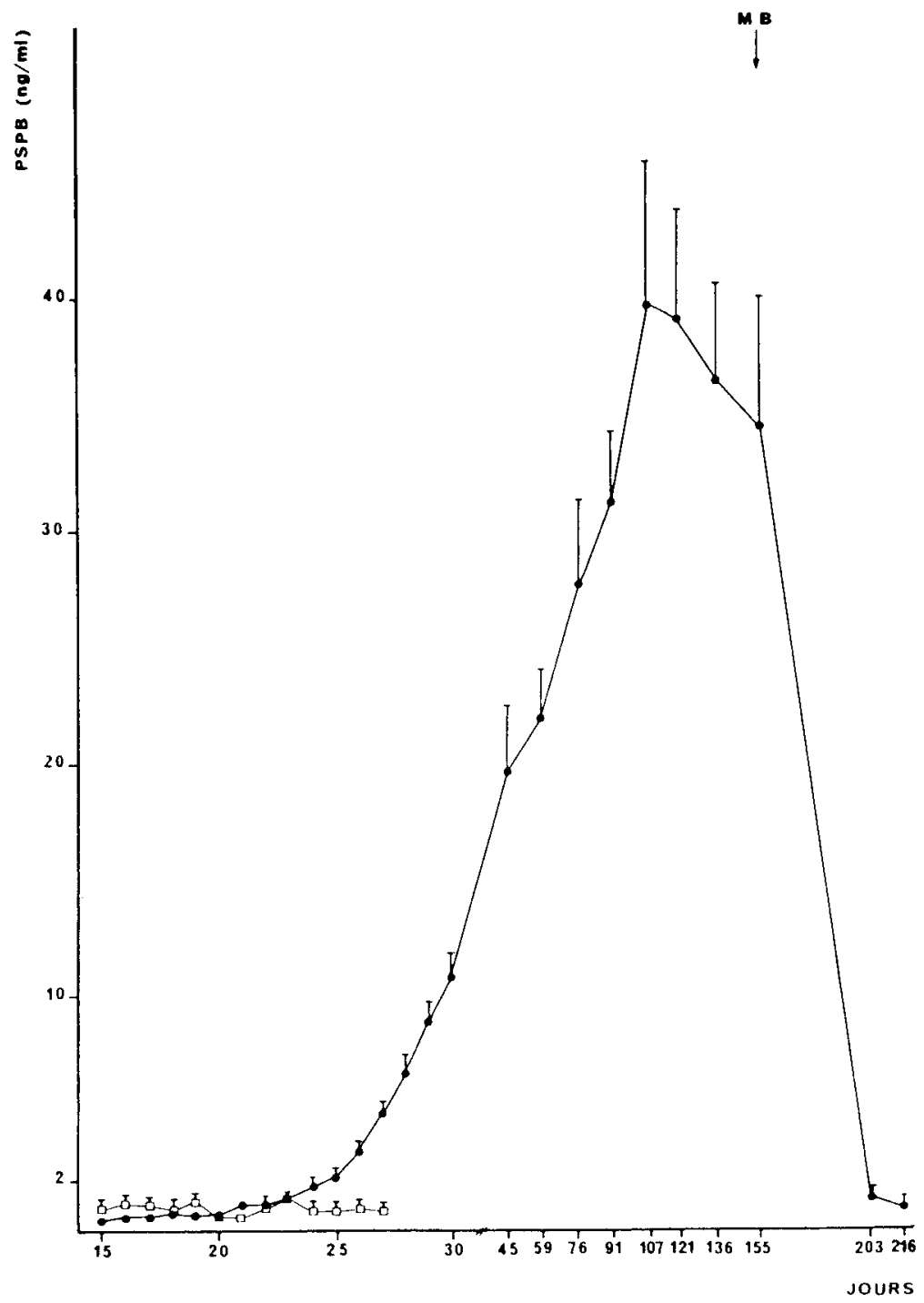

FIG. 2. - Evolution des concentrations moyennes ( \pm erreur type) de PSPB chez des chèvres gestantes $(\bullet ; \mathrm{n}=23$ ) ou non $(\square ; \mathrm{n}=7)$.

Relations entre évolution de la PSPB, mortalité embryonnaire et concentrations de progestérone.

L'utilisation du dosage de progestérone 21 à 24 jours après l'insémination a permis de séparer les cas de non-fécondation ou de mortalité embryonnaire précoce des cas de mortalité embryonnaire postérieurs au $16^{\mathrm{e}}$ jour (Humblot, 
1986). Dans le premier cas, l'insémination est suivie d'une nouvelle ovulation 21 à 24 jours plus tard et la longueur du cycle n'est pas modifiée (Northey et French, 1980 ; Humblot et Dalla Porta, 1984). En cas de mortalité embryonnaire tardive, par contre, les concentrations de progestérone plasmatique restent supérieures à $1,5 \mathrm{ng} / \mathrm{ml}$ entre le $21^{\mathrm{e}}$ et le $24^{\mathrm{e}}$ jour après l'insémination.

Deux études ont bien montré qu'en cas de mortalité embryonnaire précoce il est tout à fait exceptionnel de retrouver des concentrations de PSPB détectables (tabl. 3). En effet, ces travaux effectués dans 2 races différentes et confirmant le rôle prépondérant des mortalités embryonnaires précoces dans les échecs de gestation, ont montré que moins de $5 \%$ des femelles présentant des concentrations de progestérone faibles 21-24 jours après I'IA ont des niveaux de PSPB détectables. Cette fréquence est plus élevée (environ $30 \%$ ) chez les vaches ou génisses présentant une mortalité embryonnaire tardive avec un maintien de la fonction lutéale au-delà du $24^{\mathrm{e}}$ jour post IA. Mais chez de tels animaux la sécrétion de PSPB est déprimée par rapport aux femelles gestantes (Humblot et al., 1988a). Cet effet s'observe dès le $24^{\mathrm{e}}$ jour et l'augmentation entre J24 et J30 est plus faible qu'en cas de gestation.

TABLEAU 3

Fréquence des échecs de gestation chez des animaux présentant une élévation (PSPB +) des concentrations plasmatiques de PSPB entre 28 et 30 jours [selon Humblot et al., 1988a (1), Humblot et al., 1988c (2)].

\begin{tabular}{|c|c|c|c|c|c|c|}
\hline \multirow{3}{*}{$\begin{array}{c}\text { Expérience } \\
\text { Situation } \\
\text { après IA }\end{array}$} & \multicolumn{3}{|c|}{ (1) } & \multicolumn{3}{|c|}{ (2) } \\
\hline & \multicolumn{3}{|c|}{ Race FFPN } & \multicolumn{3}{|c|}{ Race Normande } \\
\hline & $\mathrm{N}$ & $\%$ & PSPB + & $\mathrm{N}$ & $\%$ & PSPB + \\
\hline $\begin{array}{l}\text { Non fécondatio } \\
\text { ou mortalité } \\
\text { embryonnaire } \\
\text { précoce }\end{array}$ & 63 & $35,6 \%$ & $3,1 \%(2 / 63)$ & 44 & $37 \%$ & $2,3 \%(1 / 44)$ \\
\hline $\begin{array}{l}\text { Mortalité } \\
\text { embryonnaire } \\
\text { tardive }\end{array}$ & 31 & $17,5 \%$ & $32,3 \%(10 / 31)$ & 10 & $8,4 \%$ & $30 \%(3 / 10)$ \\
\hline Gestation & 83 & $46,9 \%$ & & 65 & $54,6 \%$ & \\
\hline Total & 177 & $100 \%$ & & 119 & $100 \%$ & \\
\hline
\end{tabular}

Les données préliminaires obtenues chez la chèvre montrent que, comme chez les bovins, toutes les femelles présentant une mortalité embryonnaire tardive (lutéolyse tardive et non gestante) n'ont pas nécessairement une augmentation des concentrations de PSPB (fig. 3).

Exactitude du dosage de PSPB comme méthode de diagnostic de gestation.

Les données d'exactitude du dosage de PSPB utilisé comme méthode de diagnostic de gestation chez la vache allaitante et la vache laitière sont présentées au tableau 4. Ces travaux montrent la bonne exactitude des résultats fournis par ce dosage aussi bien pour les résultats positifs que pour les résultats négatifs. 

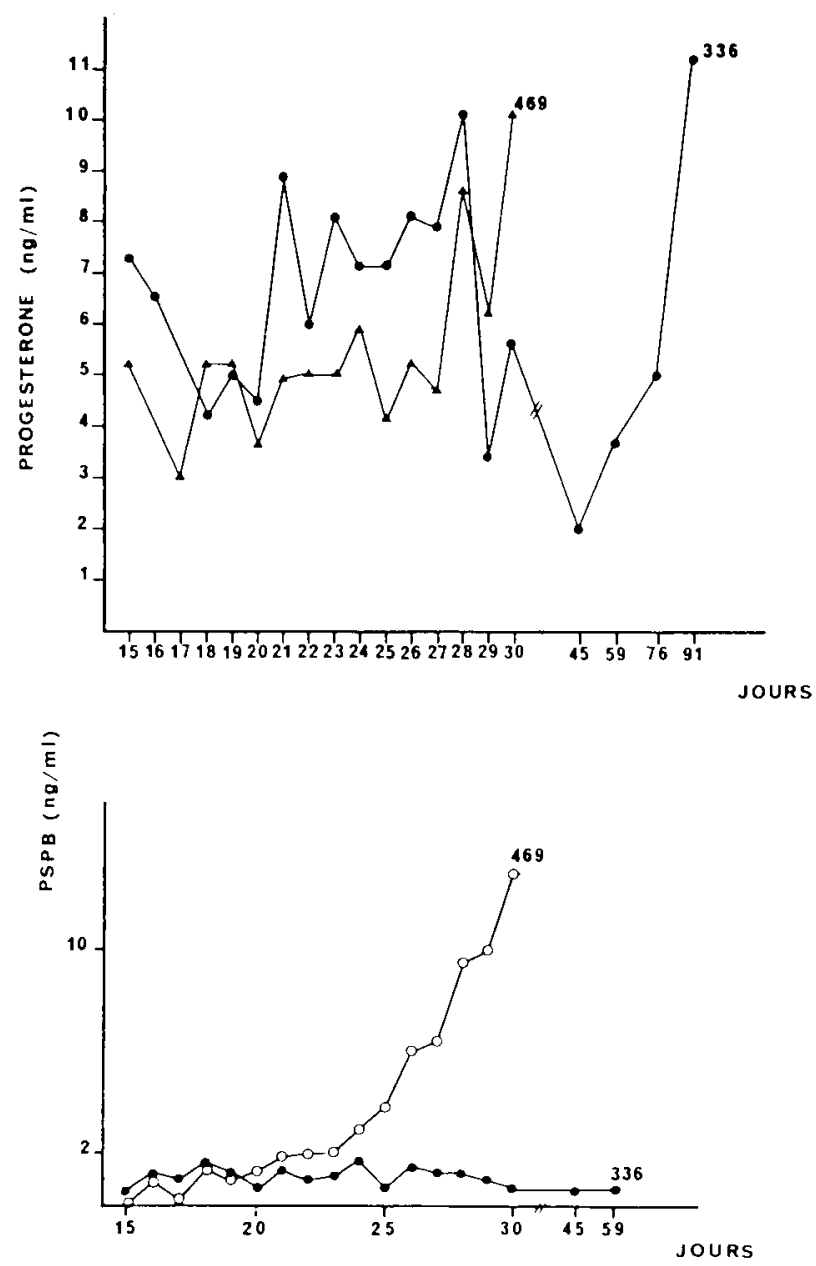

FIG. 3. - Profils individue/s de progestérone et de PSPB chez deux chèvres ayant présenté une mortalité embryonnaire tardive (selon Humblot et al., non publié).

Cependant pour obtenir une exactitude de $90 \%$ pour les résultats positifs, il convient de réaliser le test après des inséminations faites plus de 70 jours post-partum (Humblot et al., 1988b). Ceci implique que chez des vaches inséminées précocement, le prélèvement ne soit pas fait à moins de 100 jours post-partum. Enfin, la variabilité du moment d'apparition de la protéine empêche une utilisation de la méthode avant le $28^{\mathrm{e}}$ jour. En effet, dans ce cas l'exactitude des résultats négatifs diminue (Humblot et al., 1988d).

Les caractéristiques d'exactitude de cette méthode de diagnostic de gestation ont également été étudiées chez la brebis et la chèvre et sont rapportées au tableau 5. Bien qu'obtenues à partir de plus petits effectifs que chez les bovins (en 
TABLEAU 4

Exactitude du dosage de PSPB chez la vache allaitante (selon Sasser et al., 1986) et chez la vache laitière entre 28 et 35 jours (selon Humblot et al., $1988 \mathrm{~b}$ et $\mathrm{d}$ ).

\begin{tabular}{lcccc}
\hline $\begin{array}{l}\text { Type } \\
\text { d'animal }\end{array}$ & $\begin{array}{c}\text { Intervalle } \\
\text { IA/prélèvement } \\
\text { (jours) }\end{array}$ & $\mathrm{N}$ & $\begin{array}{c}\text { Exactitude } \\
\text { Résultats }+ \\
\%\end{array}$ & $\begin{array}{c}\text { Exactitude } \\
\text { Résultats }- \\
\%\end{array}$ \\
\hline Vache allaitante & $?$ & 102 & $98,8 \%(98 / 99)$ & $100 \%(3 / 3)$ \\
\hline Vache laitière & $30-35(\mathrm{~d})$ & 175 & $90,2 \%(83 / 92)$ & $100 \%(83 / 83)$ \\
\cline { 2 - 5 } & $28(\mathrm{~b})$ & 90 & $88,7 \%(55 / 62)$ & $96,4 \%(27 / 28)$ \\
\hline
\end{tabular}

TABLEAU 5

Caractéristiques d'exactitude du diagnostic de gestation par dosage de PSPB chez les ovins (selon Ruder et al., 1988) et les caprins (selon Humblot et al., non publié).

\begin{tabular}{lccccccc}
\hline & \multirow{2}{*}{$\begin{array}{c}\text { Stade de } \\
\text { gestation } \\
\text { (jours) }\end{array}$} & $\mathrm{N}$ & & \multicolumn{4}{c}{ Exactitude des diagnostics } \\
\cline { 5 - 8 } & & & $\%$ & Positifs & & \multicolumn{2}{c}{ Négatifs } \\
\hline \multirow{2}{*}{ Brebis } & $26-96$ & 33 & 93,8 & $(30 / 32)$ & 100 & $(1 / 1)$ \\
& $35-106$ & 180 & 98,8 & $(159 / 161)$ & 100 & $(19 / 19)$ \\
& $56-126$ & 33 & 96,8 & $(30 / 31)$ & 100 & $(2 / 2)$ \\
& $83-153$ & 32 & 100 & $(30 / 30)$ & 100 & $(2 / 2)$ \\
\hline Chèvre & 26 & 34 & 79,3 & $(23 / 29)$ & 100 & $(5 / 5)$ \\
\hline
\end{tabular}

particulier pour les résultats négatifs) ces données montrent que le dosage radioimmunologique de la $\mathrm{PSPB}$ même en système hétérologue (traceur et standard bPSPB ; anticorps anti-PSPB ovine) peut être utilisé à des fins de diagnostic de gestation chez les petits ruminants après le $26^{\mathrm{e}}$ jour. Avant ce stade, la variabilité dans le moment d'apparition de la protéine entraîne l'obtention de faux résultats négatifs.

En outre, dans l'étude réalisée chez les caprins, nous n'avons pas observé de différence entre l'exactitude du diagnostic par dosage de progestérone à $\mathrm{J} 21$ et celui par dosage de PSPB à J26. Ces résultats sont à confirmer à partir d'effectifs plus importants. Cependant la facilité d'utilisation du dosage de progestérone à partir du lait et la bonne exactitude des résultats obtenus avec cette méthode font que le dosage de PSPB n'apparaît réellement intéressant que lorsque la date d'IA ou de saillie n'est pas connue avec précision.

\section{Conclusion.}

Chez les ruminants où la PSPB est détectable, l'association du dosage de la PSPB avec le dosage de la progestérone est un très bon outil pour apprécier la 
chronologie des pertes embryonnaires et étudier les facteurs susceptibles de jouer un rôle sur ces événements. Chez les bovins au plan pratique, le fait que la PSPB soit sécrétée continuellement pendant la gestation et à des concentrations toujours détectables à partir du $30^{\mathrm{e}}$ jour font du dosage de cette protéine dans le sérum ou le plasma une méthode de diagnostic de gestation très souple d'utilisation dont les caractéristiques d'exactitude sont très bonnes. Elle représente donc un outil particulièrement approprié pour contrôler la reproduction chez des vaches allaitantes chez lesquelles la date exacte de saillie est souvent difficile à connaître. II est nécessaire toutefois d'utiliser cette méthode après des IA réalisées plus de 70 jours après le vêlage pour conserver une exactitude des résultats positifs proche de $90 \%$. C'est seulement dans ces conditions que l'aptitude à la détection des animaux non gestants sera optimale.

Chez les petits ruminants l'apport du dosage de PSPB par rapport au dosage de progestérone est moins grand car la fréquence des pertes embryonnaires tardives est généralement plus faible. Néanmoins cette technique apparaît intéressante dans tous les troupeaux où une confirmation de l'état de gestation à un stade quelconque du cycle de reproduction est désirée.

$27^{\prime 2}$ Réunion de la Société française pour l'Etude de la Fertilité. Paris, 29, 30 sept., $1^{\text {er }}$ oct. 1988.

\section{Références}

BUTLER J. E., HAMILTON W. C., SASSER R. G., RUDER C. A., HASS G. M., WILLIAMS R. J., 1982. Detection and partial characterization of two bovine pregnancy specific proteins. Biol. Reprod., 26, 925-933.

FISHER S. J., GIMENEZ T., HENRICKS D. M., 1985. Immunosuppressive activity associated with early pregnancy in the bovine. Biol. Reprod., 32, 894-906.

GODKIN J. D., BAZER F. W., SESSIONS F., ROBERTS R. M., 1982. Purification and properties of a major low molecular weight protein released by the trophoblast of sheep blastocysts at Days 13-21. J. Reprod. Fert., 65, 141-150.

GODKIN J. D., BAZER F. W., THATCHER W. W., ROBERTS R. M., 1984. Protein released by cultured Day 15-16 conceptuses prolong luteal maintenance when introduced into lumen of cyclic ewes. J. Reprod. Fert., 71, 57-64.

HOUSTON D. B., ROBBINS C. T., RUDER C. A., SASSER R. G., 1986. Pregnancy detection in mountain goats by assay for pregnancy specific protein B. J. Wildl. Manag., 50, 740-742.

HUMBLOT P., DALLA PORTA M. A., 1984. Effect of conceptus removal and intrauterine administration of conceptus tissus on luteal function in the cow. Reprod. Nutr. Dévelop., 24, 529-541

HUMBLOT P., 1986. La mortalité embryonnaire chez les bovins. In Recherches récentes sur fépidémiologie de la fertilité, pp. 213-242. Masson Ed. Paris.

HUMBLOT P., CAMOUS S., MARTAL J., CHARLERY J., JEANGUYOT N., THIBIER M., SASSER G., 1988a. Pregnancy specific protein B, progesterone concentrations and embryonic mortality during early pregnancy in dairy cows. J. Reprod. Fert., 83, 215-223.

HUMBLOT P., JEANGUYOT N., RUDER C. A., LERICHE I., THIBIER M., SASSER G., 1988b. Accuracy of pregnancy diagnosis by bPSPB RIA in the plasma of dairy cows 28 days after Al. In Proc. 11th Int. Congr. Anim. Reprod. Al, Dublin (Short communication). Vol. 2, p. 94 
HUMBLOT P., OLIVEIRA O., RUDER C., JEANGUYOT N., THIBIER M., SASSER R. G., 1.988c. Progesterone and PSPB concentrations at time of $\mathrm{Al}$ and at the beginning of pregnancy in the dairy cow. Proc. BARD int. Workshop on maternal recognition of pregnancy and maintenance of the corpus luteum. Jerusalem (Abstr.), p. 62.

HUMBLOT P., CAMOUS S., MARTAL J., CHARLERY J., JEANGUYOT N., THIBIER M., SASSER G., 1988d. Diagnosis of pregnancy by radioimmunoassay of a pregnancy specific protein in the plasma of dairy cows. Theriogenology, 30, 257-269.

KNICKERBOCKER J. J., THATCHER W. W., BAZER F. W., DROST M., BARON D. H., FINCHER K. B., ROBERTS R. M., 1986. Proteins secreted by Day 16 to 18 bovine conceptuses extend corpus luteum function in cows. J. Reprod. Fert., 77, $381-391$.

MARTAL J., LACROIX M. C., LOUDES C., SAUNIER M., WINTERBERGER-TORRES S., $1979 . \quad$ Trophoblastin an antiluteolytic protein in early pregnancy in sheep. J. Reprod. Fert., 56, 63-73.

NORTHEY O. L., FRENCH L. R., 1980. Effect of embryo removal and intra-uterine infusion of embryonic homogenates on the lifespan of bovine corpus luteum. J. anim. Sci., 50, 298-302.

RUDER C. A., SASSER R. G., 1986. Source of bovine pregnancy specific protein B (bPSPB) during the post-partum period and estimation of half life of bPSPB. J. anim. Sci., 63 (Suppl. 1), 335, Abstr.

RUDER C. A., SASSER R. G., DAHMEN J. J., STELLFLUG J. N., 1988. Detection of pregnancy in sheep by radioimmunoassay of sera for pregnancy specific protein B. Theriogenology (sous presse).

SASSER R. G., RUDER C. A., IVANI K. A., BUTLER J. E., HAMILTON W. C., 1986. Detection of pregnancy by radioimmunoassay of a novel pregnancy specific protein in serum of cows and a profile of serum concentrations during gestation. Biol. Reprod., 35, 936-942.

SEGERSON E. C., 1981. Immunosuppressive effect of ovine uterine secretory protein upon lymphocytes in vitro. Biol. Reprod., 25, 77-84.

SEGERSON E. C., LIBBY D. W., GETZ W. R., RANDEL R. O., 1984. Immunosuppressive effect of uterine secretory protein from angus and brahman cows upon lymphocytes in vitro. J. anim. Sci., 59, 1047-1059.

WOOD A. K., DARLING A., DUSEK G. L., SASSER R. G., RUDER C. A., 1986. Serum assays for detecting pregnancy in mule and white tailed deer. J. Wildl. Manag., 50, 684-687. 